\title{
AN ASYMPTOTIC FORMULA FOR THE DERIVATIVES OF ORTHOGONAL POLYNOMIALS ON THE UNIT CIRCLE
}

\section{CHRISTIAN HÖRUP}

1.

Let $f$ be a non-negative function and assume that $f$ and $\log f$ both belong to $L(-\pi, \pi)$. By $k$ we denote the regular analytic function in $|z|<1$, which satisfies the conditions

1) $\lim _{r \rightarrow 1-0} \operatorname{Re}\left\{k\left(r e^{i \theta}\right)\right\}=\log f(\theta)$ a.e.,

2) $k(0)$ real.

Let

$$
D(f ; z)=\exp \left\{\frac{1}{2} k(z)\right\} .
$$

The orthogonal polynomials $\Phi_{n}$ associated with $f$ are uniquely defined by

a) $(2 \pi)^{-1} \int_{-\pi}^{\pi}\left|\Phi_{n}\left(f ; e^{i \theta}\right)\right|^{2} f(\theta) d \theta=1$,

b) $\int_{-\pi}^{\pi} \Phi_{n}\left(f ; e^{i \theta}\right) \overline{\pi\left(e^{i \theta}\right)} f(\theta) d \theta=0$ for every polynomial $\pi$ of degree at most $n-1$,

c) $\varkappa_{n}(f)>0$, where $\varkappa_{n}(f)$ is the coefficient of $z^{n}$ in $\Phi_{n}(f ; z)$.

In this notation G. Szegö [3, p. 296] deduced the asymptotic formula

$$
\Phi_{n}\left(f ; e^{i \gamma}\right)=e^{i n \boldsymbol{\gamma}}\left\{D\left(f ; e^{i \boldsymbol{\gamma}}\right)\right\}^{-1}+o(1)
$$

for certain functions $f$. Later G. Freud [1] proved the same formula under simplified conditions on $f$.

The aim of this note is to derive similar formulas for the derivatives of $\Phi_{n}$ under Freud's conditions. Our method is an extension of Freud's. In the sequel we use the notations

$$
\begin{aligned}
& s_{n}(f ; a, z)=\sum_{\nu=0}^{n} \overline{\Phi_{\nu}(f ; a)} \Phi_{\nu}(f ; z), \\
& t_{n, q}(f ; a)=\sum_{\nu=0}^{n}\left|\Phi_{\nu}^{(q)}(f ; a)\right|^{2}
\end{aligned}
$$

Received December 21, 1966. 
and

$$
r_{n, q}(f ; a, z)=\sum_{\nu=0}^{n} \overline{\Phi_{\nu}(f ; a)} \Phi_{\nu}^{(q)}(f ; z)
$$

2.

Theorem. Let $f$ of period $2 \pi$ be of bounded variation, and suppose that there are constants $M$ and $m$ so that $0<m \leqq f(\theta) \leqq M$ for all $\theta$. Let $p$ be $a$ non-negative integer.

If $\gamma$ is a number such that

$$
\int_{-\pi}^{\pi} \log f(\theta) \cot \frac{1}{2}(\gamma-\theta) d \theta
$$

exists as a principal Cauchy value, then, for $z=e^{i \gamma}$, it follows that

$$
\Phi_{n}^{(p)}(f ; z)=n^{p} z^{n-p}\{\overline{D(f ; z)}\}^{-1}+o\left(n^{p}\right) .
$$

Remark. That $\Phi_{n}^{(p)}\left(f ; e^{i \theta}\right)=O\left(n^{p}\right)$ uniformly in $\theta$ and $n$ follows immediately by Bernstein's inequality and the fact $[1$, p. 286] that $\Phi_{n}\left(f ; e^{i \theta}\right)=O(1)$ uniformly in $\theta$ and $n$.

The proof of the theorem is given in sections 3-7.

3.

First we derive a suitable estimate of the difference between $\Phi_{n}^{(p)}(f ; z)$ and $\Phi_{n}^{(p)}(g ; z)$, when $f$ and $g$ satisfy the conditions of the theorem. In order to do this we use the well-known formula [3, p. 288]

$$
\varkappa_{n}(f) \bar{\Phi}_{n}(f ; z)=z^{n} s_{n}\left(f ; 0, z^{-1}\right) .
$$

Differentiating both sides with respect to $z$ we get

$$
\varkappa_{n}(f) \bar{\Phi}_{n}^{\prime}(f ; z)=n z^{n-1} s_{n}\left(f ; 0, z^{-1}\right)-z^{n-2} r_{n, 1}\left(f ; 0, z^{-1}\right),
$$

which implies the following inequality for $|z|=1$

$$
\begin{aligned}
&\left|\varkappa_{n}(f) \Phi_{n}^{\prime}(f ; z)-\varkappa_{n}(g) \Phi_{n}^{\prime}(g ; z)\right| \\
& \leqq n\left|r_{n, 0}(f ; 0, z)-r_{n, 0}(g ; 0, z)\right|+\left|r_{n, 1}(f ; 0, z)-r_{n, 1}(g ; 0, z)\right| .
\end{aligned}
$$

Differentiating (4) $p$ times we get for $|z|=1$ that

where

$$
\begin{aligned}
\mid \varkappa_{n}(f) \Phi_{n}^{(p)}(f ; z) & -\varkappa_{n}(g) \Phi_{n}^{(p)}(g ; z) \mid \\
& \leqq \sum_{\nu=0}^{n} C_{p, \nu}\left|r_{n, \nu}(f ; 0, z)-r_{n, \nu}(g ; 0, z)\right|,
\end{aligned}
$$

Math. Scand. $20-3$ 


$$
C_{p, \nu}=\left\{\begin{array}{cl}
\left(\begin{array}{l}
p \\
v
\end{array}\right)(n-v)(n-v-1) \ldots(n-p+1) & \text { if } v \leqq p-1 \\
1 & \text { if } v=p .
\end{array}\right.
$$

Later on $g^{-1}$ will be replaced by suitably selected trigonometrical polynomials.

\section{4.}

We now consider the following problem. Let $\lambda, \mu$ and $a$ be complex numbers, and let $f$ be positive and integrable on the unit circle. Determine the supremum of

$$
\left|\lambda \varrho(0)+\mu \varrho^{(q)}(a)\right|^{2}, \quad q \text { integer } \geqq 1,
$$

when $\varrho$ ranges over the set of all polynomials of degree $n$ which satisfy the condition

$$
(2 \pi)^{-1} \int_{-\pi}^{\pi} f(\theta)\left|\varrho\left(e^{i \theta}\right)\right|^{2} d \theta=1 .
$$

We shall apply a method of G. Szegö $[3$, p. 303]. Let

$$
\varrho(z)=u_{0} \Phi_{0}(f ; z)+u_{1} \Phi_{1}(f ; z)+\ldots+u_{n} \Phi_{n}(f ; z) .
$$

Then $\sum_{v=0}^{n}\left|u_{v}\right|^{2}=1$, and according to Cauchy's inequality we get

$$
\begin{aligned}
\left|\lambda \varrho(0)+\mu \varrho^{(q)}(a)\right|^{2} & =\left|\sum_{\nu=0}^{n} u_{\nu}\left\{\lambda \Phi_{\nu}(f ; 0)+\mu \Phi_{\nu}^{(q)}(f ; a)\right\}\right|^{2} \\
& \leqq \sum_{\nu=0}^{n}\left|\lambda \Phi_{\nu}(f ; 0)+\mu \Phi_{\nu}^{(q)}(f ; a)\right|^{2} \\
& =|\lambda|^{2} s_{n}(f ; 0,0)+2 \operatorname{Re}\left\{\bar{\lambda} \mu r_{n, q}(f ; 0, a)\right\}+|\mu|^{2} t_{n, q}(f ; a) .
\end{aligned}
$$

This expression is the desired maximum, since it is attained for

$$
\varrho(z)=\varepsilon\left(\sum_{\nu=0}^{n}\left|\lambda \Phi_{\nu}(f ; 0)+\mu \Phi_{\nu}^{(q)}(f ; a)\right|^{2}\right)^{-1} \sum_{\nu=0}^{n} \overline{\left\{\lambda \Phi_{\nu}(f ; 0)+\mu \Phi_{\nu}^{(q)}(f ; a)\right\}} \Phi_{\nu}(f ; z),
$$

where $|\varepsilon|=1$. If

$$
0<m \leqq f_{1}(\theta) \leqq f(\theta) \leqq f_{2}(\theta), \quad|\theta| \leqq \pi,
$$

then the preceding result shows that

$$
\begin{aligned}
|\lambda|^{2} s_{n}\left(f_{1} ; 0,0\right)+2 \operatorname{Re}\left\{\bar{\lambda} \mu r_{n, q}\left(f_{1} ; 0, a\right)\right\}+|\mu|^{2} t_{n, q}\left(f_{1} ; a\right) \\
\geqq|\lambda|^{2} s_{n}(f ; 0,0)+2 \operatorname{Re}\left\{\bar{\lambda} \mu r_{n, q}(f ; 0, a)\right\}+|\mu|^{2} t_{n, q}(f ; a) \\
\geqq|\lambda|^{2} s_{n}\left(f_{2} ; 0,0\right)+2 \operatorname{Re}\left\{\bar{\lambda} \mu r_{n, q}\left(f_{2} ; 0, a\right)\right\}+|\mu|^{2} t_{n, q}\left(f_{2} ; a\right) .
\end{aligned}
$$


For $\lambda=0$ and $\mu=1$ this reduces to

$$
t_{n, q}\left(f_{1} ; a\right) \geqq t_{n, q}(f ; a) \geqq t_{n, q}\left(f_{2} ; a\right) .
$$

From (6) we get the inequality

$$
\begin{aligned}
|\lambda|^{2}\left[s_{n}(f ; 0,0)-s_{n}\left(f_{2} ; 0,0\right)\right]+2 \operatorname{Re}\left\{\bar{\lambda} \mu\left(r_{n, q}(f ; 0, a)-r_{n, q}\left(f_{2} ; 0, a\right)\right)\right\}+ & \\
& +|\mu|^{2}\left[t_{n, q}(f ; a)-t_{n, q}\left(f_{2} ; a\right)\right] \geqq 0 .
\end{aligned}
$$

From a consideration of the discriminant of the left-hand side and an application of (7) it follows that

$$
\begin{aligned}
& \left|r_{n, q}(f ; 0, a)-r_{n, q}\left(f_{2} ; 0, a\right)\right|^{2} \\
& \leqq\left\{s_{n}(f ; 0,0)-s_{n}\left(f_{2} ; 0,0\right)\right\}\left\{t_{n, q}\left(f_{1} ; a\right)-t_{n, q}\left(f_{2} ; a\right)\right\} .
\end{aligned}
$$

Since $s_{n}(f ; 0,0)=\left(\varkappa_{n}(f)\right)^{2}$ (cf. e.g. [3, p. 288]) we get

$$
\begin{aligned}
\left|r_{n, q}(f ; 0, a)-r_{n, q}\left(f_{2} ; 0, a\right)\right|^{2} & \\
& \leqq\left\{\left(\varkappa_{n}(f)\right)^{2}-\left(\varkappa_{n}\left(f_{2}\right)\right)^{2}\right\}\left\{t_{n, q}\left(f_{1} ; a\right)-t_{n, q}\left(f_{2} ; a\right)\right\} .
\end{aligned}
$$

5.

We next want an estimate of the right side in (8) where $f_{1}$ and $f_{2}$ are replaced by reciprocals of suitable trigonometrical polynomials. If $f$ satisfies the conditions of the theorem, $F(\theta)=f^{-1}(\theta)$ is clearly of bounded variation. Thus it follows from theorem 1 in [2] that there exists a sequence of trigonometrical polynomials $\left(W_{n}\right)$ (where $W_{n}$ is of degree at most $n$ ) such that

and

$$
2 m^{-1} \geqq W_{n}(\theta) \geqq F(\theta)=f^{-1}(\theta)
$$

$$
\int_{-\pi}^{\pi}\left|W_{n}(\theta)-F(\theta)\right| d \theta=O\left(n^{-1}\right) .
$$

Condition (2) in our theorem implies that $F$ is continuous at $\gamma$. Hence the remark on theorem 1 in $[2, \mathrm{p} .281]$ shows that to every positive number $\varepsilon$ there exist numbers $\delta_{1}$ and $N_{1}$ such that

$$
\left|W_{n}(\theta)-F(\gamma)\right|<\varepsilon
$$

for all $\theta$ and $n$ satisfying $|\theta-\gamma|<\delta_{1}$ and $n>N_{1}$.

Similarly another sequence of trigonometrical polynomials $\left(w_{n}\right)$ exists satisfying

and

$$
F(\theta) \geqq w_{n}(\theta) \geqq \frac{1}{2} M^{-1}>0
$$

$$
\int_{-\pi}^{\pi}\left|w_{n}(\theta)-F(\theta)\right| d \theta=O\left(n^{-1}\right) .
$$


To every positive number $\varepsilon$ there exist numbers $\delta_{2}$ and $N_{2}$ such that

$$
\left|w_{n}(\theta)-F(\gamma)\right|<\varepsilon
$$

for all $\theta$ and $n$ satisfying $|\theta-\gamma|<\delta_{2}$ and $n>N_{2}$. Furthermore we know according to $[1, \mathrm{p} .287]$ that

$$
\left[\varkappa_{n}(f)\right]^{2}-\left[\varkappa_{n}\left(w_{n}^{-1}\right)\right]^{2}=O\left(n^{-1}\right) .
$$

In order to get an estimate of

$$
t_{n, q}\left(W_{n}^{-1} ; e^{i \gamma}\right)-t_{n, q}\left(w_{n}^{-1} ; e^{i \gamma}\right),
$$

which is the second factor of the right side of (8) with $f_{1}$ and $f_{2}$ replaced by $W_{n}{ }^{-1}$ and $w_{n}{ }^{-1}$ respectively, we proceed as follows.

With the chosen $\varepsilon$ and with $\delta=\min \left(\delta_{1}, \delta_{2}\right)$ and $N=\max \left(N_{1}, N_{2}\right)$, we consider two trigonometrical polynomials $\chi$ and $\psi$ satisfying the conditions (12) and (13):

$$
\begin{array}{ll}
F(\gamma)+2 \varepsilon \geqq \chi(\theta), & \theta \in\left(\gamma-\frac{1}{2} \delta, \gamma+\frac{1}{2} \delta\right), \\
F(\gamma)+\varepsilon \leqq \chi(\theta), & \theta \in(\gamma-\delta, \gamma+\delta), \\
3 m^{-1} \geqq \chi(\theta) \geqq 2 m^{-1}, & \theta \notin(\gamma-\delta, \gamma+\delta), \\
F(\gamma)-2 \varepsilon \leqq \psi(\theta), & \theta \in\left(\gamma-\frac{1}{2} \delta, \gamma+\frac{1}{2} \delta\right), \\
F(\gamma)-\varepsilon \geqq \psi(\theta), & \theta \in(\gamma-\delta, \gamma+\delta), \\
\frac{1}{3} M^{-1} \leqq \psi(\theta) \leqq \frac{1}{2} M^{-1}, & \theta \notin(\gamma-\delta, \gamma+\delta) .
\end{array}
$$

Because of (9) and (10)

$$
\chi(\theta) \geqq W_{n}(\theta) \geqq w_{n}(\theta) \geqq \psi(\theta), \quad n>N .
$$

By (7) it then follows that

$$
t_{n, q}\left(\chi^{-1} ; e^{i \gamma}\right) \geqq t_{n, q}\left(W_{n}^{-1} ; e^{i \gamma}\right) \geqq t_{n, q}\left(w_{n}^{-1} ; e^{i \gamma}\right) \geqq t_{n, q}\left(\psi^{-1} ; e^{i \gamma}\right) .
$$

Since $\psi$ is a positive trigonometrical polynomial, $D\left(\psi^{-1} ; z\right)$ is a polynomial of the same type [3, p.287]. We denote this polynomial by $h\left(\psi^{-1} ; z\right)$ and by $[3, \mathrm{p} .287]$ we have

$$
\Phi_{n}\left(\psi^{-1} ; z\right)=z^{n} \bar{h}\left(\psi^{-1} ; z^{-1}\right)
$$

for all $n \geqq$ degree of $\psi$. Differentiating both sides $q$ times with respect to $z=e^{i \theta}$ we get

$$
\Phi_{n}^{(q)}\left(\psi^{-1} ; z\right)=n^{q} z^{n-q} \bar{h}\left(\psi^{-1} ; z^{-1}\right)+O\left(n^{q-1}\right) .
$$

Since $\psi(\theta)=\left|h\left(\psi^{-1} ; e^{i \theta}\right)\right|^{2}$, we get $(\theta=\gamma)$

and hence

$$
\left|\Phi_{n}^{(q)}\left(\psi^{-1} ; e^{i \gamma}\right)\right|^{2}=n^{2 q} \psi(\gamma)+O\left(n^{2 q-1}\right)
$$




$$
t_{n, q}\left(\psi^{-1} ; e^{i \gamma}\right)=\psi(\gamma) \sum_{0}^{n} \nu^{2 q}+O\left(n^{2 q}\right)=\psi(\gamma)\left\{n^{2 q+1} /(2 q+1)+O\left(n^{2 q}\right)\right\} .
$$

Similarly we get

$$
t_{n, q}\left(\chi^{-1} ; e^{i \gamma}\right)=\chi(\gamma)\left\{n^{2 q+1} /(2 q+1)+O\left(n^{2 q}\right)\right\} .
$$

Introducing the last two estimates in (14) we get by aid of (12a) and (13a) that

$$
\begin{aligned}
\left(f^{-1}(\gamma)+3 \varepsilon\right) n^{2 q+1} /(2 q+1) & \geqq t_{n, q}\left(W_{n}^{-1} ; e^{i \gamma}\right) \\
& \geqq t_{n, q}\left(w_{n}^{-1} ; e^{i \gamma}\right) \geqq\left(f^{-1}(\gamma)-3 \varepsilon\right) n^{2 q+1} /(2 q+1)
\end{aligned}
$$

if $n$ is sufficiently large. This yields

$$
t_{n, q}\left(W_{n}{ }^{-1} ; e^{i \gamma}\right)-t_{n, q}\left(w_{n}{ }^{-1} ; e^{i \gamma}\right)=o\left(n^{2 q+1}\right) .
$$

By (8) and (11) we find that

$$
r_{n, q}\left(f ; 0, e^{i \gamma}\right)-r_{n, q}\left(w_{n}{ }^{-1} ; 0, e^{i \gamma}\right)=o\left(n^{q}\right) .
$$

In (5) we replace $g^{-1}$ by $w_{n}$ and $z$ by $e^{i \gamma}$. Since according to [1]

$$
s_{n}\left(f ; 0, e^{i \gamma}\right)-s_{n}\left(w_{n}^{-1} ; 0, e^{i \gamma}\right)=o(1),
$$

we see that every term in the sum $(5)$ is $o\left(n^{p}\right)$. It follows that

$$
\varkappa_{n}(f) \Phi_{n}^{(p)}\left(f ; e^{i \gamma}\right)-\varkappa_{n}\left(w_{n}^{-1}\right) \Phi_{n}^{(p)}\left(w_{n}^{-1} ; e^{i \gamma}\right)=o\left(n^{p}\right) .
$$

6.

Next we want to prove that

$$
\Phi_{n}^{(p)}\left(w_{n}^{-1} ; e^{i \gamma}\right)=n^{p} e^{i(n-p) y} \overline{\left\{D\left(w_{n}^{-1} ; e^{i \gamma}\right)\right\}^{-1}}+o\left(n^{p}\right) .
$$

The formula

$$
\Phi_{n}\left(w_{n}{ }^{-1} ; e^{i \theta}\right)=e^{i n \theta} \overline{\left\{D\left(w_{n}^{-1} ; e^{i \theta}\right)\right\}^{-1}}
$$

follows in the same way as (15) from [3, p. 287]. Differentiation of this equation with respect to $\theta$ gives

$$
\begin{aligned}
\frac{d^{p}}{d \theta^{p}} \Phi_{n}\left(w_{n}^{-1} ; e^{i \theta}\right)= & (i n)^{p} e^{i n \theta} \overline{\left\{D\left(w_{n}^{-1} ; e^{i \theta}\right)\right\}^{-1}}+ \\
& +\sum_{v=1}^{p}\left(\begin{array}{c}
p \\
v
\end{array}\right)(i n)^{p-v} e^{i n \theta} \frac{d^{v}}{d \theta^{v}}\left\{D\left(w_{n}^{-1} ; e^{i \theta}\right) w_{n}(\theta)\right\}
\end{aligned}
$$

since $\overline{\left\{\mathrm{D}\left(w_{n}{ }^{-1} ; e^{i \theta}\right)\right\}^{-1}}=D\left(w_{n}^{-1} ; e^{i \theta}\right) w_{n}(\theta)$.

In the sequel we shall use the following lemma.

Lemma. Let $\left(T_{n}\right)$ be a sequence of trigonometric polynomials of degree $n$. If there are constant $\varepsilon, \delta$ and $M$ such that 
then

$$
\begin{array}{ll}
\left|T_{n}(\theta)\right| \leqq M & \text { for all } \theta, \\
\left|T_{n}(\theta)\right| \leqq \varepsilon & \text { for }|\theta| \leqq \delta,
\end{array}
$$

$$
\left|T_{n}{ }^{\prime}(\theta)\right| \leqq 8 n \varepsilon \quad \text { for }|\theta| \leqq \frac{1}{2} \delta \text { and } n>N,
$$

where $N$ depends on $\varepsilon, \delta$ and $M$.

Proof. For $|\theta| \leqq \frac{1}{2} \delta$ our assumptions imply

The expression

$$
\frac{T_{n}(\theta+h)-T_{n}(\theta-h)}{\sin h} \leqq \begin{cases}4|h|^{-1} \varepsilon & \text { for }|h| \leqq \frac{1}{2} \delta, \\ 8 M \delta^{-1} & \text { for }|h|>\frac{1}{2} \delta .\end{cases}
$$

$$
u_{n}(h)=\left[T_{n}(\theta+h)-T_{n}(\theta-h)\right][\sin h]^{-1}
$$

is a trigonometrical polynomial in $h$ of degree at most $n$. By Bernstein's inequality, $\left|u_{n}{ }^{\prime}\right| \leqq n \max \left|u_{n}\right|$, it follows that in an interval of length $n^{-1}$ with center at a maximum point of $\left|u_{n}\right|$, the absolute value $\left|u_{n}\right|$ is not less than half the maximum. By aid of this observation we get that

$$
\left|\frac{T_{n}(\theta+h)-T_{n}(\theta-h)}{\sin h}\right| \leqq \max \left(16 n \varepsilon, 8 M \delta^{-1}\right) \text { for }|\theta| \leqq \frac{1}{2} \delta,
$$

which for $n$ sufficiently large is $\leqq 16 n \varepsilon$. This implies that

$$
\left|T_{n}{ }^{\prime}(\theta)\right| \leqq 8 \cdot n \cdot \varepsilon \quad \text { for }|\theta| \leqq \frac{1}{2} \delta \text { and } n>N,
$$

where $N$ only depends on $\varepsilon, \delta$ and $M$. The proof of the lemma is finished.

The definition of $\left\{w_{n}\right\}$ shows that $\left\{\left|w_{n}(\theta)\right|\right\}$ is bounded and furthermore we know according to (9) that to every positive $\varepsilon$ there exist numbers $\delta$ and $N_{0}$ such that

$$
\left|w_{n}(\theta)-F(\gamma)\right|<\varepsilon
$$

when $|\theta-\gamma| \leqq \delta$ and $n>N_{0}$. Thus by our lemma

$$
\left|w_{n}{ }^{\prime}(\theta)\right| \leqq 8 n \varepsilon
$$

when $|\theta-\gamma| \leqq \frac{1}{2} \delta$ and $n$ is sufficiently large. We find by a $q$-fold iteration that

$$
\left|w_{n}^{(q)}(\theta)\right|<(8 n)^{q} \varepsilon \quad \text { for }|\theta-\gamma| \leqq 2^{-q} \delta .
$$

From the definition of $D$ we obtain

$$
\begin{aligned}
\frac{d}{d \theta} D\left(w_{n}{ }^{-1} ; e^{i \theta}\right)=- & \frac{1}{2} D\left(w_{n}{ }^{-1} ; e^{i \theta}\right) \\
& \cdot\left\{\frac{w_{n}{ }^{\prime}(\theta)}{w_{n}(\theta)}+\frac{i}{2 \pi} \int_{-\pi}^{\pi} \frac{d}{d t}\left[\log w_{n}(t)\right] \cot \frac{1}{2}(\theta-t) d t\right\} .
\end{aligned}
$$


We know that there exists a constant $c>0$ such that $c^{-1}<w_{n}(\theta)<c$ for all sufficiently large $n$. Thus, it follows from

$$
\left|D\left(w_{n} ; e^{i \theta}\right)\right|^{2}=w_{n}(\theta)
$$

that $D\left(w_{n} ; e^{i \theta}\right)$ satisfies a similar inequality.

To obtain an estimate of the integral in the last term in (20) for $|\theta-\gamma| \leqq \frac{1}{4} \delta$ and sufficiently large $n$ we proceed as follows. Putting $n^{-1} \varepsilon^{\frac{1}{2}}=\eta$ and combining the estimates of the proof of the lemma with (19), we find for one part of the integral in (20) that

$$
\begin{aligned}
& \left|\int_{\theta-\eta}^{\theta+\eta} w_{n}{ }^{\prime}(t) \cot \frac{1}{2}(\theta-t) d t\right|=\left|\int_{\theta-\eta}^{\theta+\eta}\left(\frac{w_{n}{ }^{\prime}(t)}{w_{n}(t)}-\frac{w_{n}{ }^{\prime}(\theta)}{w_{n}(\theta)}\right) \cot \frac{1}{2}(\theta-t) d t\right| \\
& \leqq \int_{\theta-\eta}^{\theta+\eta}\left|\frac{w_{n}{ }^{\prime}(t)-w_{n}{ }^{\prime}(\theta)}{w_{n}(t)} \cot \frac{1}{2}(\theta-t)\right| d t+\int_{\theta-\eta}^{\theta+\eta}\left|\frac{w_{n}{ }^{\prime}(\theta)}{w_{n}(\theta)} \frac{w_{n}(\theta)-w_{n}(t)}{w_{n}(t)} \cot \frac{1}{2}(\theta-t)\right| d t
\end{aligned}
$$

$\leqq 2 M 16 n 8 n \varepsilon 2 n^{-1} \varepsilon^{-\frac{1}{1}}+4 M^{2} 8 n 16 n \varepsilon^{2} 2 n^{-1} \varepsilon^{-1}=\varepsilon^{\frac{1}{2}} O(n)$.

For the integral on the rest of the circle we have the estimate

$$
\begin{aligned}
\int_{\theta+\eta}^{2 \pi+\theta-\eta} \frac{d}{d t} & {\left[\log w_{n}(t)\right] \cot \frac{1}{2}(\theta-t) d t } \\
& =\left[\log w_{n}(t) \cot \frac{1}{2}(\theta-t)\right]_{\theta+\eta}^{2 \pi+\theta-\eta}+\frac{1}{2} \int_{\theta+\eta}^{2 \pi+\theta-\eta} \log w_{n}(t)\left[\sin \frac{1}{2}(\theta-t)\right]^{-2} d t \\
& =\varepsilon^{\ddagger} O(n) .
\end{aligned}
$$

Thus

$$
\left|\frac{d}{d \theta} D\left(w_{n}{ }^{-1} ; e^{i \theta}\right)\right| \leqq \varepsilon^{1} O(n) \quad \text { for }|\theta-\gamma| \leqq 1 \delta .
$$

If we differentiate (20) $q-1$ times and make repeated use of our lemma as above, we find that

$$
\left|\frac{d^{q}}{d \theta^{q}} D\left(w_{n}^{-1} ; e^{i \theta}\right)\right| \leqq \varepsilon^{\frac{1}{1}} O\left(n^{q}\right) \text { for }|\theta-\gamma| \leqq 2^{-q-1} \delta
$$

and hence

$$
\frac{d^{q}}{d \theta^{q}} D\left(w_{n}^{-1} ; e^{i \gamma}\right)=o\left(n^{q}\right)
$$

Then (17) follows by applying (19) and (21) to (18). 
7.

Finally using the following two results $[1$, p. 287, 289]:

$$
0<\mu \leqq x_{n}\left(w_{n}{ }^{-1}\right) \leqq \lambda,
$$

where $\mu$ and $\lambda$ are constants not depending on $n$, and

$$
\lim _{n \rightarrow \infty} D\left(w_{n}^{-1} ; e^{i \gamma}\right)=D\left(f ; e^{i \gamma}\right),
$$

we obtain the desired result in the following way. Applying (11) and (22) to (16), we get

$$
\Phi_{n}^{(p)}\left(f ; e^{i \gamma}\right)=\Phi_{n}^{(p)}\left(w_{n}^{-1} ; e^{i \gamma}\right)+o\left(n^{p}\right),
$$

and we find from (17) that this can be written in the form

$$
\Phi_{n}^{(p)}\left(f ; e^{i \gamma}\right)=n^{p} e^{i(n-p) \gamma}\left\{\overline{\left.D\left(w_{n}^{-1} ; e^{i \gamma}\right)\right\}^{-1}}+o\left(n^{p}\right) .\right.
$$

Now the statement (3) follows by applying (23). The theorem is proved.

\section{REFERENCES}

1. G. Freud, Eine Bemerkung zur asymptotischen Darstellung von Orthogonalpolynomen, Math. Scand. 5 (1957), 285-290.

2. G. Freud and T. Ganelius, Some remarks on one-sided approximation, Math. Scand. 5 (1957), 276-284.

3. G. Szegö, Orthogonal polynomials (Amer. Math. Soc. Coll. Publ. 23), New York, 1959.

UNIVERSITY OF GÖTEBORG, SWEDEN 